ESTUDIOS

\title{
La algoritmización del trabajo en la Cuarta Revolución Industrial
}

\author{
Labour algorithmization in the Fourth Industrial Revolution
}

\author{
Helena Emerick Abaurre (ip) \\ Universidade Federal do Espírito Santo, Brasil \\ Ricardo José Macedo de Britto Pereira \\ Centro Universitário do Distrito Federal, Brasil \\ Cláudio Jannotti da Rocha \\ Universidade Federal do Espírito Santo, Brasil
}

RESUMEN En medio de la Cuarta Revolución Industrial y de la gig economy, los algoritmos deciden quién trabajará, cuándo, cómo y por cuánto. La creación de perfiles, las funciones de gestión de recursos humanos, la recomendación de contenido y los sistemas de revisión son impulsados por la inteligencia artificial y los macrodatos, creando un ciberespacio laboral dirigido por un jefe algorítmico. En este contexto son dos las preguntas centrales de la investigación: ¿cuál es la interacción entre los algoritmos y el trabajo?, y ¿por qué medios es posible coordinar este proceso disruptivo? Se emplea la metodología cualitativa desde una perspectiva deductiva, plasmada en la investigación bibliográfica y documental. La hipótesis a probar es que la algoritmización laboral ocurre junto con la tendencia a la plataformización laboral, que se ve potenciada por la ciberización del medio ambiente de trabajo.

PALABRAS CLAVE Cuarta Revolución Industrial, derecho laboral, ciberización, algoritmos, plataformas digitales.

ABSTRACT Amid the Fourth Industrial Revolution and the gig economy, it is the algorithm that decides who will work, when, how and for how much. Profiling, human resource management functions, content recommendation and review systems are powered by artificial intelligence and big data, creating a labour cyberspace led by an algorithmic boss. In this context, it is assigned central issues of the research: how is la- 
bour algorithmized? By which means is it possible to address this disruptive process? To answer these questions, qualitative methodology is employed from a deductive perspective, embodied in international bibliographic and documentary research. The hypothesis to be tested is that the labour algorithmization occurs along with the labour platformization tendency, which is enhanced by the cyberization of labour environment.

KEYWORDS Fourth Industrial Revolution, labour law, cyberization, algorithms, digital platforms.

\section{Introducción}

El universo laboral influye en la sociedad y es influenciado por ella, lo que lleva a un proceso de transformación constante. El mundo laboral cambia como lo hace la humanidad todos los días; se transforma a medida que mujeres y hombres desarrollan nuevos deseos, nuevas necesidades y nuevos proyectos. Una de las transmutaciones más claras en el ámbito laboral se refiere al impacto de la máquina y de la tecnología en el trabajo. Si bien algunas profesiones tienden a desaparecer debido a la automatización y el alto empleo de la inteligencia artificial y el internet de las cosas, lo que preocupa a trabajadores manuales y artesanos, otros profesionales dependen en gran medida de las plataformas digitales para obtener su sustento diario.

La Cuarta Revolución Industrial recién ha experimentado sus primeros años. Sin embargo, al mismo tiempo, los conductores de aplicaciones, mensajeros, moderadores de contenido, gamers, crowdworkers, pinners, podcasters, memers, youtubers, influencers y bloggers ya son algunas categorías de trabajadores que tienen el ciberespacio como su entorno laboral. Donde el internet de las cosas posibilita la fusión de software y hardware, los motores, el carbón y los uniformes típicos de la línea de producción fordista dan lugar a elementos disruptivos como algoritmos, likes y características de aprendizaje automático. En este escenario, rápidamente se advierte la transmutación socioambiental laboral: se asiste al surgimiento de un (no tan admirable) mundo nuevo dentro de un mundo viejo.

La evolución de la interacción entre el ser humano en su medio ambiente laboral y la tecnología ha sido descrita por la Organización Internacional del Trabajo (OIT) a partir de los modos de empleo de instrumentos telemáticos, lo que permite identificar tres generaciones, a saber: la oficina en casa (el home office), la oficina móvil y la oficina virtual (OIT, 2019).

La primera generación se desarrolló a mediados de los años setenta. Los estudios demuestran que la etapa inicial de la modalidad estaba restringida al home office, con lugares de trabajo remoto dentro de las casas de los empleados, los cuales tenían un perfil barato y ecológico, una vez que se reducían los costos y polución generados por los traslados. Entretanto, se trataba aún de una interacción estacionaria, vez 
que, en aquel momento histórico, ni siquiera las tecnologías de última generación permitieron la ejecución de un trabajo deslocalizado, ya que los medios telemáticos dependían de un lugar de base fijo para funcionar. Los instrumentos telemáticos eran indisociables de tomadas, servidores, monitores y estaciones fijas de conexión a cables físicos de internet.

La segunda etapa de la interacción evolutiva se distingue de la primera por mejorar las tecnologías de información y comunicación en dispositivos inalámbricos, más pequeños y livianos, como computadores portátiles y teléfonos celulares, que posibilitaron que los empleados trabajaran no solo en casa, sino desde casi cualquier lugar. El núcleo del trabajo a distancia, en este sentido, pasa a ser la parcialidad del trabajo presencial en las instalaciones del empleador y la posibilidad de sustituir el trabajo presencial por el mediado remotamente por los instrumentos tecnológicos. Así, aunque la percepción del teletrabajo se mantuvo en gran parte relacionada con la oficina en casa, la oficina móvil rompió con la división biespacial del trabajo, de tal manera que se reconoció gradualmente que el trabajo no estaba necesariamente conectado a un espacio físico y que se podía realizar «aquí, allá, en cualquier momento y en cualquier lugar» (OIT, 2019: 7).

En este camino evolutivo, se reveló en la tercera generación la posibilidad de que el trabajo no se realice en un lugar específico, sino en constante movimiento debido a los teléfonos inteligentes. A diferencia de la generación anterior - en la cual el trabajo pasó a ser móvil, pero la información aún necesitaba ser transportada en expedientes físicos, con una marcada separación entre las tecnologías de la información y la comunicación-, en la etapa actual de interacción del trabajo y las tecnologías los dispositivos informativos y comunicativos se condensan en nubes de tal manera que solo requieren una pequeña unidad de almacenamiento físico (microchips de memoria y red de datos móviles) que permite que todo el universo de trabajo esté literalmente en manos del trabajador, un ciberespacio al que se llama oficina virtual.

Esta oficina virtual accesible a través de dispositivos portátiles se describe como una forma de trabajo completamente nueva y emergente. Se observa la interacción resultante de cinco décadas de evolución iniciada por el deseo inicial de reducir los costos de los desplazamientos, que implicó la movilización del trabajo de oficina y que en el momento presente se ha expandido a prácticamente todos los aspectos posibles de la vida humana. Así, se establece un elemento de ubicuidad en la rutina laboral, ya que a partir del hecho de que un trabajador traiga consigo su teléfono inteligente se vuelve posible acceder a vías de información y comunicación casi desde cualquier espacio-tiempo en contextos urbanos (avenidas, transporte público, ascensores).

Actualmente, la interacción entre labor y la tecnología se inserta en el contexto de la Cuarta Revolución Industrial. El mundo del trabajo está siendo testigo de la fusión de sistemas digitales, físicos y biológicos provocada por la sinergia y convergencia de múltiples innovaciones. Ocurre que, como marco de innovación y transmutación de 
quizá una cuarta generación, no tan solo el teletrabajo, sino que modalidades variables de empleo - incluso el tradicional- se reconocen sujetas a la comunicación sistémica de los artificios de la industria 4.0, como una orquestra sinfónica comandada por un director algorítmico.

Según un informe elaborado por el Foro Económico Mundial (FEM) intitulado Diversity, equity and inclusion 4.o, el comienzo de la década ha llamado la atención sobre las intersecciones entre las siguientes tendencias principales: la aceleración de la Cuarta Revolución Industrial, las disrupciones del mercado laboral en relación con la adaptabilidad al trabajo digital y un amplio llamado a una mayor inclusión, equidad y justicia social (FEM, 2019: 11). Las disrupciones, más tarde o más temprano, transformarán múltiples aspectos del mercado laboral; se advierte que «a largo plazo, ningún sector de la economía quedará fuera del alcance de los algoritmos» (Prassl, 2019: 8). De hecho, se prevé la plena absorción por las oficinas virtuales de los artificios habituales de la industria 4.o, en particular, el internet de las cosas, la computación en nubes, los macrodatos, el análisis de datos y la inteligencia artificial.

Para comprender mejor la intersección de las tecnologías, aclaramos que el internet de las cosas comprende la creciente variedad de dispositivos conectados a la red mundial de computadoras, como sensores, medidores, chips de identificación de radiofrecuencia y otros dispositivos que están integrados en varios objetos cotidianos que les permiten enviar y recibir varios tipos de datos. Los dispositivos pueden variar desde computadoras, teléfonos, televisores, pantallas, automóviles, transportes o cualquier cosa que pueda conectarse a internet, incluyendo ropas, embalajes y paquetes. De hecho, se prevé que la cantidad de conexiones de internet de las cosas alcance la marca de trillones de sensores digitales en 2025, incluidas las posibilidades de la tecnología usable, como en el caso de trabajadores cuyos uniformes o relojes contienen artificios digitales que permiten el envío de informaciones en tiempo real al empleador (Schwab, 2020: 139).

Además, la tecnología inalámbrica de banda ancha móvil de quinta generación - conocida popularmente por la abreviación $5 \mathrm{G}$ - es fundamental para optimizar el ambiente del internet de las cosas, una vez que ofrece mayor capacidad para manejar volúmenes masivos de datos y con ello la posibilidad de conectar muchos más dispositivos, creando un entorno laboral ciberespacial no solo rodeado, sino impulsado y casi que indisociable de los artificios alimentados por las condiciones digitales.

La computación en la nube permite el acceso a programas, archivos y servicios a través de internet, sin la necesidad de instalar programas o almacenar datos, de ahí la alusión a la «nube». En otras palabras, consiste en el suministro de servicios informáticos (incluidos servidores, almacenamiento, bases de datos, redes, software, análisis e inteligencia) a través de internet («la nube»), cuyo objetivo es ofrecer una innovación más rápida, recursos flexibles y economías de escala.

La tecnología hace posible que se acceda a archivos y se realicen diferentes tareas 
a través de internet, sin necesidad de instalar aplicaciones en la computadora. El almacenamiento de datos se realiza en servicios en línea, a través de una red. Desde cualquier computadora y en cualquier lugar, se puede acceder a información, archivos y programas en un solo sistema, ya que los datos no se guardan en el disco duro de ninguna computadora, sino que están disponibles en la web.

A su vez, los desarrollos en la inteligencia artificial, incluido el aprendizaje automático (machine learning), son avances tecnológicos que permiten que los sistemas simulen una inteligencia similar a la humana, yendo más allá de la programación de órdenes específicas para tomar decisiones de forma autónoma, basándose en patrones de datos para generar conocimientos y predecir el comportamiento utilizando algoritmos.

Los macrodatos, por su parte, se refieren a datos que son tan grandes, rápidos o complejos que es imposible procesarlos con métodos tradicionales, pero se requieren técnicas de análisis de datos. Se caracteriza por las tres «V»: volumen, velocidad y variedad. Las organizaciones recopilan datos de una variedad de fuentes que varían desde dispositivos inteligentes inseridos en el contexto del internet de las cosas hasta equipos industriales, redes sociales y plataformas. Esta recopilación se produce a una velocidad sin precedentes y los datos vienen de todo tipo, color y forma.

Por lo tanto, se instalan cuestiones epicentrales de la presente investigación: ¿Cuál es la interacción entre los algoritmos y el trabajo? ¿Por qué medios es posible coordinar este proceso disruptivo? Para responder a estas preguntas se emplea la metodología cualitativa desde una perspectiva deductiva, plasmada en la investigación bibliográfica y documental, respaldada por la doctrina internacional y en las publicaciones de organismos internacionales. De acuerdo a este método, la hipótesis a probar es que la algoritmización laboral ocurre junto con la virtualización individual y social, potenciada por la ciberización que posibilita la plataformización laboral. La percepción alcanzada es que la protección de la alta implementación de algoritmos en el ambiente laboral requiere rescatar los orígenes del derecho laboral y fortalecer sus fundamentos teóricos para que las garantías de los trabajadores en el ciberespacio estén aseguradas.

Amoldados a esta premisa, analizaremos primeramente cómo se desarrolla el funcionamiento algorítmico con vistas a comprender su absorción por la industria 4.o. En seguida, se explorará la interacción entre los algoritmos y su empleo en la dinámica del trabajo en la economía gig. Avanzando en la exposición, serán revisadas las consideraciones sobre la opacidad y la dependencia algorítmica observadas en el ciberespacio laboral, sobre todo en las nuevas modalidades de trabajo, como el, así llamado, trabajo colaborativo en línea (crowdworking) y el trabajo bajo demanda.

Objetivando el análisis crítico, finalmente será debatido cómo coordinar soluciones favorables a los trabajadores que se ven bajo los mandos algorítmicos. Se defiende que derramar la luz del derecho bajo la opacidad de la algoritmización laboral 
requiere esfuerzos colectivos, para que el trabajo en la gig economy se realice en un ciberespacio equilibrado, justo, seguro y no excluyente.

\section{Comprensión del funcionamiento algorítmico}

Aunque se vincule casi automáticamente al ámbito de la tecnología de la información, el concepto de algoritmo es tan antiguo como las matemáticas y, por lo tanto, anterior e independiente de la digitalización contemporánea y la existencia de cualquier computadora, disco duro u otro sustrato físico sobre el cual puedan implementarse (Sandvig y otros, 2016: 5). Los algoritmos se manifiestan desde los inicios de la civilización egipcia, cuando se diseñaban fórmulas para resolver problemas cotidianos como la próxima inundación del río Nilo, por ejemplo.

En palabras simples, el algoritmo es una serie de instrucciones secuenciales que permiten ejecutar acciones o programas. Su alta implantación en las más diversas actividades cotidianas hoy en día se debe a la suma de tres factores principales, todos ellos potenciados continuamente en el centro de la Cuarta Revolución Industrial. En primer lugar, la expansión de la capacidad de procesamiento de las computadoras, que aceleró la ejecución de tareas complejas; en segundo lugar, la llegada de los macrodatos, es decir el almacenamiento barato de cantidades gigantescas de información en la dinámica descrita por la ley de Moore; ${ }^{1}$ y en tercer lugar, la tecnología de aprendizaje automático, más conocida como machine learning, que permite recoger y transformar datos aislados en aspectos del mundo jamás cuantificados.

A través de la tecnología de aprendizaje automático, los algoritmos pueden crear y modificar otros algoritmos, mejorando exponencialmente la capacidad de las máquinas para resolver varios problemas procedimentales que forman parte de la rutina diaria del trabajo, lo que representa un vínculo autoalimentado y duradero entre las máquinas, los humanos y el software. Como afirman distintos estudiosos, el desarrollo de máquinas inteligentes cambia las reglas del juego porque cada algoritmo tiene sus respectivos inputs y outputs: los datos ingresan a la computadora, el algoritmo hace lo que su código determina con esos datos y luego sale el resultado. El aprendizaje automático cambia esta lógica: tanto los datos como el resultado deseado ingresan a la máquina y el producto es un algoritmo capaz de hacer verdadera la relación entre los datos y el resultado. Los algoritmos inteligentes, también conocidos como «aprendices», son algoritmos que crean otros algoritmos. Con el aprendizaje automá-

1. De acuerdo con ella la velocidad de los procesadores y el número de transmisores en una unidad central de procesamiento se duplica cada dos años; este aumento continuo en la capacidad de procesamiento va unido a la caída en los precios del hardware e incide en una interacción aún mayor y más rápida entre dispositivos y macrodatos. Esta combinación dio a los algoritmos la posibilidad de identificar, en las más diversas actividades, todo tipo de patrones imperceptibles para el ojo humano. Al respecto, véase Schwab (2016: 29). 
tico, las computadoras escriben sus programas, para que nosotros no tengamos que hacerlo (Schertel y Mattiuzzo, 2019: 45).

A medida que se aumenta la disponibilidad de conjuntos de datos y la capacidad de la tecnología de aprendizaje automático, mayor es el poder otorgado a los algoritmos para mediar nuestra experiencia del mundo y mayor es su capacidad de sustituir a los humanos en la toma de decisiones. Los algoritmos «inteligentes» actúan no solo para mejorar los procesos automatizados y maximizar las estrategias comerciales, sino que también para crear formas de interacción subjetiva profunda que no se habían pensado antes y que implican complejos análisis en la elaboración de perfiles: bajo la óptica de los macro datos pueden evaluar características, personalidad, inclinaciones y propensiones de una persona, como su orientación sexual, estados emocionales, opiniones políticas y personales, su capacidad y habilidad para trabajos, funciones y encargos específicos o incluso su predisposición a enfermedades crónicas, entre otros aspectos.

La operación del aprendizaje automático se basa en la evolución constante y la redefinición de parámetros: el control algorítmico ya no se limita a las experiencias enseñadas a través de conjuntos de datos de entrenamiento y rutinas analíticas programadas en adelante (Prassl, 2019: 17). Frank Pasquale, autor del notable libro The black box society, aborda el problema desde el punto de vista de que la autoridad se expresa algorítmicamente, ya que las decisiones antes basadas en la reflexión humana ahora son automáticas. Esta mala práctica se traduce en estructuras opacas en las que los inputs y outputs no son conocidos con precisión (Pasquale, 2015: 12) y, aun así, se emplean cada vez más masivamente en la dinámica laboral. La falta de transparencia en los algoritmos es una preocupación real con respecto a las consecuencias de su implementación en el mercado laboral de la gig economy, como se detalla en las siguientes secciones.

\section{Los algoritmos y la dinámica laboral en la gig economy}

En el contexto de la Cuarta Revolución Industrial, y específicamente de la gig economy, los algoritmos se pueden implementar en la dinámica laboral desde el momento de la contratación, pasando por el seguimiento de las actividades de los trabajadores hasta la rescisión del contrato de empleo. El impacto es aún mayor en las nuevas modalidades de trabajo en las que todavía no se reconoce el vínculo laboral —si bien la relación de empleo se caracteriza de hecho-, como ocurre con los trabajadores en plataformas digitales, ya sea que las arquitecturas virtuales estén estructuradas para el trabajo colaborativo en línea o para el trabajo bajo demanda.

Como actualmente vivimos en un contexto de economía digital, las categorías de trabajadores digitales han recibido especial atención de organismos internacionales como la Comisión Europea, la Conferencia de las Naciones Unidas sobre Comercio y 
Desarrollo (Unctad) , la Organización para la Cooperación y el Desarrollo Económi$\cos (\mathrm{OCDE})$, y la OIT, que han elaborado, respectivamente, el informe "Impact of the digital transformation on EU labour markets»; el informe «Digital economy report 2019.Value creation and capture: Implications for developing countries»; ${ }^{3}$ el documento «Broadband policies for Latin America and the Caribbean: A digital economy toolkit»; ${ }^{4} \mathrm{y}$ el documento «Digital labour platforms and the future of work: Towards decent work in the online world». En todos los informes publicados por estos organismos internacionales se reconocen los cambios que la tecnología promueve en la economía global en su conjunto.

La tecnología puede cambiar tanto el proceso de búsqueda de candidatos para un empleo como el de contratación y también facilitar las operaciones de gestión del rendimiento y de recursos humanos: desde el acompañamiento de la localización en tiempo real de los trabajadores a la creación de perfiles - como se denomina a la práctica de profiling-, los algoritmos de la gig economy son capaces de conectar a empleadores, consumidores, plataformas, aplicaciones y sitios web. Estas conexiones pueden derivar en contratos formales o informales para realizar actividades puramente en línea - como el oficio de los trabajadores digitales que crean contenido para las redes sociales virtuales - o actividades en vivo fuera de línea, esto es, en el mundo real, o incluso regímenes híbridos que mezclan actividades en línea y presenciales. Además, los algoritmos pueden localizar el trayecto desde el lugar de trabajo hasta la residencia de los empleados, optimizando la explotación de los trabajadores, como se verifica en los viajes de los motoristas y repartidores de aplicaciones (OIT, 2018: 4).

En cuanto a las prácticas de contratación, las empresas de tecnología están ofreciendo programas de software que automatizan el análisis de currículos, clasificando candidatos, realizando ofertas y determinando niveles salariales. En un primer momento, el abandono de los procesos selectivos tradicionales por parte de las grandes empresas tecnológicas tuvo como finalidad diversificar el grupo de candidatos concurrentes a puestos de trabajo. En cambio, la literatura académica afirma que los riesgos permanecieron presentes en los procesos de reclutamiento basados en algoritmos, lo que generó consecuencias poco éticas.

Aunque la utilización de la inteligencia artificial tenga el objetivo de minimizar

\footnotetext{
2. Comisión Europea, 8 de abril de 2019, disponible en https://bit.ly/32kv6Nd.

3. Conferencia de las Naciones Unidas sobre Comercio y Desarrollo, 4 de septiembre de 2019, disponible en https://bit.ly/3Z7AD5G.

4. Organización para la Cooperación y el Desarrollo Económicos, 21 de junio de 2016, disponible en https://bit.ly/3FwqjX4.

5. Organización Internacional del Trabajo, 20 de septiembre de 2018, disponible en https://bit. ly/2Z19aEE.
} 
las discriminaciones en la ocasión de contratación, la complicación que acompaña al artificio es que las personas cuyas características no coinciden con las representadas en las entradas de la base de datos utilizada para entrenar el algoritmo - los inputsson tendencialmente excluidas de los outputs. No se clasifican estos determinados candidatos para los puestos, una vez que el recurso algorítmico entiende que su candidatura es menos atractiva.

Uno de los ejemplos más notorios de la negligencia algorítmica ocurrió a principios de 2019, cuando la herramienta de inteligencia artificial de Amazon se notició declaradamente sexista. El software de inteligencia fue creado en 2014 como un medio para clasificar el currículo y seleccionar automáticamente a los candidatos más talentosos o adecuados para las funciones específicas de cargos. Sin embargo, el sistema se capacitó en una base de datos construida con respaldo en los resultados de las candidaturas durante los diez años anteriores, esto es, en resultados de procesos selectivos cuyas contrataciones fueron mayoritariamente hombres. Por lo tanto, el recurso automatizado manifestó un aprendizaje sesgado y no neutral en cuanto al género: el algoritmo se enseñó rápidamente a favorecer a los candidatos masculinos sobre los femeninos, penalizando a los currículos que incluían la palabra mujer $\mathrm{y}$, según se informa, rebajando la calificación de las graduadas de universidades exclusivamente femeninas.

La llamada «herramienta de inteligencia artificial sexista» de Amazon pone el foco sobre las preocupaciones acerca de qué tan confiable y no discriminatoria puede ser la utilización de algoritmos en procesos selectivos. A medida que Silicon Valley desarrolla artificios basados en el aprendizaje automático, las empresas deben considerar el riesgo de que los algoritmos puedan estar sesgados, insertando sexismo, racismo, homofobia o xenofobia al reproducir prejuicios profundamente arraigados en códigos computacionales. Es de esta manera que implícitamente se destacan las disparidades de la sociedad en sus procesos internos automatizados, como cuando los solicitantes de empleo con nombres que suenan blancos, como Emily, recibieron $50 \%$ más de devoluciones de llamada que aquellos con nombres que suenan afroamericanos, como Lakisha, por ejemplo.

Aparte de eso, es igualmente preocupante, en particular, que el software de análisis de la fuerza laboral - a lo que se llama workforce analytics-sustituya el poder del empleador de monitorear a los empleados, sancionarlos y terminar la relación laboral. La gestión por algoritmos es una práctica pionera en la gig economy, surgiendo como un recurso para detectar y prevenir que los trabajadores «jueguen» a sistemas como las plataformas de Uber y de Amazon. Las empresas tienen como característica común la posesión de mecanismos de desactivación, avisos y notificaciones de terminación para cuando los conductores rechazan tareas (entregas, viaje y rutas) no rentables, o cuando la evaluación de sus servicios se apunta por debajo de un índice deseado de productividad y satisfacción en la actividad. 
El académico Jeremias Prassl (2019) postula que automatizar las decisiones del empleador a través de artificios de codificación es un punto de inflexión revolucionario para la gestión de recursos humanos basada en datos. En medio de este proceso, las personas y las emociones se convierten en números, correos electrónicos, identidades virtuales y plataformas. Un proceso que puede ser denominado de ludificación, como si se tratara de un videojuego con diversos niveles, misiones y desafíos: así es la vida de un trabajador bajo la labor algorítmica.

La ascensión del análisis de personas dinamizada por los algoritmos - a lo que se denomina people analytics - se ilustra perfectamente en la dinámica típica de la gig economy, retratada en la operación de las plataformas digitales. El modus operandi de las arquitecturas digitales destinadas al trabajo colaborativo en línea y al trabajo bajo demanda tienen como dínamos y propulsores los mecanismos de recomendación, revisión, reputación y calificación para administrar y evaluar su fuerza laboral. El empleo del trabajador «uberizado» se mantiene o se termina si el algoritmo entiende suficientes la cantidad de estrellas que recolectó a lo largo de los viajes y entregas realizadas.

Sin embargo, el potencial de automatizar las decisiones de gestión a través de la inteligencia artificial abarca todas las dimensiones del trabajo, incluido el espectro socioeconómico completo de los lugares de trabajo, así como todo el ciclo de vida de la relación laboral (Prassl, 2019: 3). Si los empleadores durante el taylorismo y el fordismo alegaban que uno de los «puntos débiles» de los sistemas era el costo de medir el desempeño de los trabajadores individuales, hoy, en la industria 4.o, los empleadores pueden contar con los costos marginales mínimos para monitorear a los empleados, «socios conductores» y usuarios, ejerciendo su poder directivo de innumerables formas y creando así un ejército de trabajadores socialmente excluidos que recurren a las plataformas digitales como una última alternativa de sostenerse a través del hope labour o trabajo de esperanza. Este último término se usa para referirse a trabajos no remunerados o mal remunerados que se realizan, generalmente, con el objetivo de obtener exposición o experiencia y con la esperanza de que conduzcan a un trabajo futuro (Mackenzie y McKinlay, 2020).

En este contexto del trabajo de esperanza por intermedio de las plataformas digitales, verificamos que el jefe algorítmico es un Panoptes contemporáneo, una figura mitológica que todos ven, pero que permanece invisible. Al enfrentarse a denuncias de despidos en represalia a la actividad sindical concertada en uno de sus almacenes, Amazon reveló el uso extensivo de la gestión algorítmica: el demandante había sido despedido por falta de productividad, según lo determinado por un algoritmo no parcial. La administración del almacén local afirmó en la defensa de la empresa que no tenía acceso, control o comprensión de los detalles del sistema implementado (Prassl, 2019: 15). 
En la gig economy, los algoritmos son los artificios que definen los ingresos financieros de los trabajadores. El trabajo a través de plataformas digitales surge en la segunda década del siglo XXI como una modalidad de servicios caracterizada por la dependencia de medios electrónicos en línea (plataformas, aplicaciones y sitios web) que permiten el desarrollo de las actividades de la empresa y de los trabajadores, conectando a las personas con los proveedores de servicios (Rodríguez-Piñero, 2020: 148).

Los trabajadores de la gig economy viven un momento similar al vivido por los trabajadores de las industrias del siglo XIX, cuando se produce el cambio en la organización del trabajo de la casa a la fábrica por la necesidad de utilizar tecnologías sobre las que los trabajadores no tenían propiedad. De este éxodo surgieron los primeros reclamos laborales, ya que los dueños de la industria $-\mathrm{y}$ de la tecnología indispensable para el trabajo- se encontraban en una condición privilegiada que les permitía abusar de los trabajadores. En la Cuarta Revolución Industrial la reorganización del trabajo en plataformas profundiza aún más la necesidad de los trabajadores de recurrir a las herramientas de los empleadores - antiguamente la maquinaria de fábrica, y, ahora, las redes sociales virtuales, las plataformas de diferentes naturalezas y sus respectivos algoritmos-, lo que les permite producir, aunque de manera dependiente y subordinada a los dictados de los poseedores de la inteligencia operacional algorítmica.

En efecto, se constata una relación de ultradependencia, una vez considerado que, además de revertir la ajenidad de los riesgos del trabajo - por la cual la carga es exclusiva del empresario y del empleador-, la vida de los trabajadores se deriva únicamente de cómo se mide su relevancia en la World Wide Web. Es el número basado en algoritmos de revisiones, recomendaciones, premios alcanzados, entregas completadas y nuevos seguidores, entre otras características disruptivas, lo que define los ingresos de los trabajadores. Un algoritmo también decide si un trabajador «uberizado» realizará entregas a las once de la mañana o a las tres de la madrugada, desde el atardecer hasta el amanecer. No es el conductor ni el entregador quien organiza sus actividades, puesto que el jefe algorítmico designa rutas seguras y rentables a los trabajadores, o determina si es preciso que se queden horas en atascos producto de la atracción que generan las tarifas dinámicas de sus aplicaciones.

El algoritmo hace visible el servicio que el trabajador ofrece a las multitudes conectadas en diversas plataformas y también define la aparición de las publicaciones de los creadores de contenido digital en las líneas de feeds y stories de redes sociales virtuales de los usuarios consumidores; así es como conecta a las partes potencialmente interesadas. A esta notable capacidad de control se suma que los riesgos se trasladan a la espalda del trabajador como si fuesen una carga indebida que casi se materializa en los paquetes que los «uberizados» llevan a diario. 
La implementación de algoritmos en la dinámica laboral de la gig economy es un problema en sí mismo, ya que gana aún más fuerza y musculatura cuando se integra con el internet de las cosas, promoviendo el modelo disruptivo de la conexión entre el trabajador de la plataforma y el usuario-cliente estructurando el trabajo colaborativo en línea y el trabajo bajo demanda. En el trabajo bajo demanda y los contratos de cero horas, el trabajo se asigna a sujetos específicos a través de un proceso algorítmico que analiza las ubicaciones de las partes. Esta dinámica algorítmica basada en la ubicación está presente en las plataformas de transporte (99, Uber, Lyft, Cabify), de entrega (Deliveroo, Uber Eats, iFood, Rappi, Shipp, Loggi), de servicios domésticos (Care. com, Diaríssima) y de microtareas (Task Rabitt, Get Ninjas, Colmeia).

El trabajo colaborativo en línea, o crowdwork, a su vez, se caracteriza por ser una actividad intermitente promovida por la computación en la nube y que se realiza independientemente de la ubicación de los trabajadores. Por tanto, el servicio se puede ofrecer de dos formas. En primer lugar, a trabajadores específicos, como ocurre con la industria de creación de contenido digital (por ejemplo, influencers, bloggers, vloggers, memers), autónomos (por ejemplo, Upwork) y moderadores de contenido. En segundo lugar, como ofertas para el público, como sucede en el caso de las microtareas (por ejemplo, Amazon Mechanical Turk, Clickworker) y el trabajo en público basado en concursos (por ejemplo, 99 diseños).

Según estudios desarrollados por la Comisión de la Unión Europea, desde 2010 se ha producido una consolidación de la economía de la inteligencia artificial y de la industria 4.0, cuya característica es la interacción entre el internet de las cosas, los macrodatos, el análisis de datos y la computación ubicua, que logrará en 2050 la creación de un ecosistema artificialmente inteligente. ${ }^{6}$ A partir de la percepción de dependencia en general de las operaciones algorítmicas en este nuevo orden económico, la mano invisible del mercado idealizada por Adam Smith pasa a ser digital, algorítmica y dirigida por los actores de Silicon Valley.7 Sin embargo, como se establece en la Declaración de Filadelfia, el trabajo no es una mercancía; desde la perspectiva de la legislación laboral, el tema se vuelve realmente problemático y requerirá acción correctiva cuando la opacidad de los inputs de los procesos automatizados conduzca a resultados prejudiciales, incorporando sesgos poco éticos en el trabajo en el ciberespacio.

Es en este contexto que surgen los oligopolios, constituidos por empresas trans-

6. Véase «Final report of the High-Level Expert Group on the impact of the digital transformation on EU labour markets», Comisión de la Unión Europea, 8 de abril de 2019, p. 33, disponible en https://bit. ly/3kKJLHs.

7. Véase «Informe sobre la economía digital 2019 - Creación y captura de Valor: repercusión para los países en desarrollo», Conferencia de las Naciones Unidas sobre Comercio y Desarrollo, 4 de septiembre de 2019, p. 109, disponible en https://bit.ly/2Z2Dfns. 
nacionales que se organizan en redes y a través del capital especulativo y, por tanto, operan en las lógicas compuestas por la tríada internet, globalización y ciberización, construyendo un imperio de bits y bytes que se encajan de acuerdo con los intereses del mercado.

\section{Opacidad y dependencia algorítmica}

La falta de transparencia en las operaciones algorítmicas agrega aún más niveles de dificultad al problema: las únicas percepciones que llegan a los ojos de los usuarios y trabajadores son los outputs de los algoritmos. Se desconoce cómo se diseñó un algoritmo específico, quién lo programó, qué datos ayudaron a construirlo o cuáles son los pasos en que funciona. Estas condiciones de opacidad están bien ilustradas por la metáfora de Platón del anillo de Giges y por su alegoría de la cueva. A las grandes empresas tecnológicas se les otorga la inteligibilidad del funcionamiento algorítmico, quedando las compañías exentas de la responsabilidad por los daños de los procesos automatizados, como si llevaran un manto de invisibilidad que les exime de sus actos. Al mismo tiempo, los usuarios y trabajadores de la plataforma se limitan a mirar las sombras producidas por los resultados algorítmicos, como si estuvieran encadenados, vez que incapaces de comprender completamente cómo se lograron los outputs y sin ninguna posibilidad de reacción (Pasquale, 2015: 199).

Hay innumerables ejemplos de episodios en los que los algoritmos produjeron resultados poco éticos y en los que los trabajadores perjudicados por decisiones automatizadas no pudieron hacer nada al respecto de los daños. Algunos de ellos son: la herramienta de inteligencia artificial sexista de Amazon; la supresión disimulada de influencers negras en las redes sociales virtuales; la desmonetización arbitraria de videos en Youtube; la discriminación contra afroamericanos en procesos de selección automatizados; los cierres automáticos de controladores de aplicaciones; la brecha salarial de género entre trabajadores colectivos, entre otros (Emerick, Jannotti y Vasconcelos, 2021).

Ignorar el potencial destructivo de los algoritmos para la dinámica laboral es banalizar el mal, en palabras de Hannah Arendt; el daño causado por resultados algorítmicos sesgados puede deteriorar todo el ciberespacio de trabajo, precisamente porque se propaga como un hongo en la superficie de las plataformas digitales y en sistemas de inteligencia artificial para la gestión de empleados. Si bien los recursos están (no por mucho tiempo) desprovistos de conciencia y no hacen más que cumplir las órdenes que les encomendó el programador, la banalidad del mal se expresa precisamente en la ausencia del pensamiento de quien lo practica.

$\mathrm{Si}$ en el pasado los trabajadores autónomos no necesitaban inversiones particulares en tecnología para vender sus servicios de manera competitiva en el mercado, ahora, por el contrario, deben necesariamente comprender el funcionamiento de las 
plataformas digitales — sus algoritmos, tasas de participación, alcance de las publicaciones, tarifas dinámicas, sistemas de recomendación, selección y monetizaciónpara ser contratables, empleados y visibles en las pantallas de la gig economy.

\section{Derramando la luz del derecho bajo la opacidad de la algoritmización laboral}

Una vez expuesto cómo se desenvuelve la algoritmización laboral en la Cuarta Revolución Industrial, pasamos ahora a la pregunta acerca de los medios de protección a los trabajadores en este proceso disruptivo. Al mismo tiempo que el algoritmo recuerda la idea del Panoptes que inspiró las obras de Foucault, también podemos comparar el funcionamiento de los algoritmos inteligentes dinamizados por el aprendizaje automático con la criatura mitológica hebrea del gólem: un ser místico creado artificialmente por la humanidad para servirle, cuya fuerza y poder crece día a día y que, inconsciente de ello, si no es bien comandado es capaz de destruir al amo (o programador) cuyas ordenes antes seguía.

Debemos recordar, en este contexto, que la tecnología se desarrolla para ayudar al hombre, no para subordinarlo ni perjudicarlo. No se trata de un elemento estático, sino de uno de constante dinamismo que obliga a los actores sociales a actuar atentamente a favor de los derechos de los trabajadores frente a las variables tecnológicas. La inercia de los operadores de derecho frente al avance de las tecnologías corre el riesgo de volver anacrónica la orden jurídica, restando al derecho la capacidad de observar los intereses de la persona humana en evolución frente a la velocidad característica del aprendizaje automático y de los demás artificios tecnológicos (Doneda, 2019: 54).

La academia internacional aborda posibles soluciones en el campo de la informática y de la ciencia de la computación para controlar el potencial destructivo probado de los algoritmos y no convertirlo en un gólem imparable impulsado por los macrodatos. Algunas de ellas son, por ejemplo, la inserción de datos de formación más representativos; la codificación inclusiva, que ofrece igualdad de oportunidades por diseño; y la formación de equipos de tecnología de la información más diversos y representativos, capaces de identificar sesgos algorítmicos, de modo que la tecnología opere de manera no excluyente.

Sin embargo, analizando el problema desde la perspectiva de la algoritmización del trabajo, es decir, desde la perspectiva del derecho del trabajo, se hace evidente que arrojar la luz del derecho bajo la opacidad algorítmica requiere esfuerzos conjuntos de colectivos de trabajadores, empresarios, abogados, entes gubernamentales, y de la magistratura laboral para que el trabajo en la gig economy se realice en un ciberespacio equilibrado, justo, seguro y libre de prejuicios.

Por mucho que el algoritmo sea efectivo debido a la tecnología del aprendiza- 
je automático, el recurso sigue siendo una creación inconsciente del genio humano cuya operación debe ser monitoreada cuidadosamente por él. En este contexto, debe destacarse el deber de las empresas de incluir en sus programas de integridad estructuras de cumplimiento normativo que garanticen la conformidad de las decisiones automatizadas con el ordenamiento jurídico. En el caso brasileño, las prácticas comerciales deben reflexionar no solo sobre la integración de la Constitución Federal, la Consolidación de las Leyes Laborales (CLT) ${ }^{8}$ y las normas infraconstitucionales específicas, sino también acerca del cumplimiento de la Ley General de Protección de Datos (LGPD) ${ }^{9}$ y de los tratados y convenios internacionales, en particular los de la OIT, ratificados por Brasil.

Aunque el dispositivo de la Ley General de Protección de Datos que preveía la revisión humana de decisiones automatizadas mediadas por algoritmos fue objeto de objeción presidencial en 2019, la legislación específica se guía por la aplicación de los principios descritos en su artículo sexto. ${ }^{10}$ Por ello, particularmente en cumplimiento de los principios de transparencia, prevención y no discriminación, las empresas deben organizar sus programas de integridad para evitar que las decisiones automatizadas produzcan resultados poco éticos, así como para ofrecer estructuras transparentes que permitan comprender cómo se manejaron sus datos a los trabajadores que se sientan obstaculizados por una decisión tomada en una forma exclusivamente automatizada. Las empresas deben asegurarse de antemano que la automatización de los procesos internos, ya sea de contratación, supervisión o cese de la relación de trabajo, se ajusten al ordenamiento jurídico, no quedando excluidas de esta obligación, obviamente, las empresas que operan las plataformas digitales, las cuales proporcionan actualmente la subsistencia de cuatro millones de trabajadores en Brasil. ${ }^{11}$

Si fracasa la prevención a través de las políticas de cumplimiento de normativa, la fiscalización realizada por el servicio gubernamental de persecución laboral, el poder judiciario del trabajo y por los abogados en general, puede hacer uso - como prevé el artículo octavo de la CLT $-{ }^{12}$ de jurisprudencia y precedentes extranjeros en

8. Decreto Ley 5.452, 1 de mayo de 1943, disponible en https://bit.ly/3HTV 3 D1.

9. Ley 13.709, 14 de agosto de 2018, disponible en https://bit.ly/30Dupph.

10. De la misma Ley 13.709: «Artículo 6 Las actividades de tratamiento de datos personales deberán respetar la buena fe y los siguientes principios: [...] VI, transparencia: garantía, a los titulares, de información clara, y de fácil acceso sobre el tratamiento y los respectivos agentes de tratamiento, observados los secretos comerciales e industriales; VIII, prevención: adopción de medidas para prevenir la ocurrencia de daños debido al procesamiento de datos personales; IX, no discriminación: imposibilidad de realizar un tratamiento con fines discriminatorios ilícitos o abusivos». La traducción es propia.

11. Véase «Apps como Uber e iFood se tornam "maior empregador" do Brasil», Exame, 28 de abril de 2019, disponible en https://bit.ly/3wS1yBe.

12. Véase Decreto Ley 5.452: «Artículo 8. Las autoridades administrativas y la Justicia del Trabajo, a falta de disposiciones legales o contractuales, resolverán, en su caso, por jurisprudencia, por analogía, por 
un ejercicio de derecho comparado, apegándose, principalmente, a los estándares internacionales de derechos humanos.

Los estudios de derecho comparado demuestran que ya existen discusiones sobre el término algorithmic responsibility. Los congresistas de Estados Unidos están analizando el proyecto del Algorithmic Accountability Act, por el cual las organizaciones del sector privado deberán evaluar si los algoritmos que impulsan sus sistemas de inteligencia artificial son parciales o discriminatorios. De manera similar, en abril de este año la Unión Europea emitió pautas para el uso ético de los recursos de aprendizaje automático, estableciendo medidas que responsabilizan a las empresas por cualquier consecuencia social del uso de inteligencia artificial.

Sin duda, la valoración de los efectos de las rutinas algorítmicas automatizadas también puede ser sometida al control de la convencionalidad. Algunos de los tratados internacionales de derechos humanos ratificados por Brasil que se destacan por prohibir la discriminación — incluso la provocada por algoritmos- son: la Declaración Universal de Derechos Humanos de 1948 (artículo 2); el Pacto Internacional de Derechos Civiles y Políticos de 1966 (artículo 26); el Pacto Internacional de Derechos Económicos, Sociales y Culturales de 1966 (artículos 2 y 7); la Declaración relativa a los fines y objetivos de la Organización Internacional del Trabajo (Declaración de Filadelfia), anexa a la Constitución de la OIT; la Declaración de la OIT relativa a los principios y derechos fundamentales en el trabajo de 1998; el Convenio 111 de la OIT sobre discriminación en materia de empleo y ocupación de 1958; la Declaración Americana de Derechos y Deberes del Hombre de 1948; la Convención Americana sobre Derechos Humanos de 1969 y el Protocolo de San Salvador, de la Organización de los Estados Americanos.

De hecho, después de todo, la matriz y epicentro del derecho laboral siempre ha sido, es y siempre será el principio protector.

\section{Consideraciones finales}

La algoritmización laboral se desarrolla por la ciberización del entorno laboral promovida en medio de la Cuarta Revolución Industrial. Ya sea en las relaciones laborales tradicionales o en las nuevas modalidades de trabajo que surgen en el contexto de la gig economy y de la economía de la inteligencia artificial, los algoritmos se han demostrado omnipresentes y omniscientes.

En este artículo se analizó la transmutación del ambiente laboral a partir de los impactos algorítmicos que se hicieron posibles por la integración entre las tecnolo-

equidad y demás principios generales y normas de derecho, especialmente el laboral y, aun así, según usos y costumbres, derecho comparado, pero siempre de tal forma que ningún interés de clase o privado prevalezca sobre el interés público». La traducción es propia. 
gías de la inteligencia artificial, el internet de las cosas, la computación en nubes y los macrodatos.

Inicialmente, se demostró que los desarrollos de las tecnologías en el bulto de la Cuarta Revolución Industrial hacen que el derecho y el mundo del trabajo vivencie la fusión sinérgica y en convergencia de sistemas digitales, físicos y biológicos, en una gama variada de elementos disruptivos. Se detallaron las bases del funcionamiento algorítmico y se analizaron las transformaciones provocadas en este modus operandi por la intersección entre el aprendizaje automático y los macrodatos, específicamente el surgimiento de «algoritmos inteligentes».

Posteriormente, se presentó cómo la opacidad de los procesos automatizados algorítmicamente es perjudicial para los trabajadores, sean los tradicionales obreros empleados o los muchos independientes participes de la masa de gig workers que recurren al trabajo colaborativo en línea (crowdwork) o al trabajo bajo demanda. Se verificó al largo de la bibliografía internacional, así como a partir de informes de organismos internacionales que la implementación de los algoritmos en la dinámica laboral es constante e ininterrumpida, siendo los algoritmos los «jefes» que deciden elementos estructurales de las actividades como son los horarios, la remuneración, la productividad esperada e incluso la continuidad del vínculo de trabajo o el despido.

Se indicó que en muchas de las nuevas profesiones que surgieron a partir de la Cuarta Revolución Industrial ocurre una dependencia algorítmica potencialmente dañosa a las garantías fundamentales del trabajo.

Por último, a partir de la consciencia de la urgencia de derramarse la luz del derecho bajo la opacidad de la algoritmización laboral, se ha indicado cómo las estructuras legales de los sujetos sociales deben ocuparse de promover la integración de las prácticas y rutinas de la algoritmización laboral a todo el ordenamiento jurídico nacional e internacional. Se verificó que es solamente a partir de acciones colectivas y conjuntas que se hará posible la creación de soluciones para la transformación de un ciberespacio laboral justo. Por lo tanto, corresponde a los encargados de hacer cumplir la legislación del trabajo saber interpretar los instrumentos normativos para que sean efectivos en el mundo del trabajo contemporáneo, incluso contra daños automatizados.

De manera imperativa, los intérpretes del derecho laboral no deben permitir que la algoritmización laboral lleve a la clase trabajadora a una especie de inframundo, a una nueva dimensión de excluidos y de prejuiciados. En efecto, hay que promover la igualdad de oportunidades digitales, garantizando a todos un mercado laboral plural y combativo de cualquier forma de discriminación. 


\section{Referencias}

Alessi, Cristina (2020) «Trabajo en plataforma y no discriminación en el derecho de la EU». En Macarena Hernández Bejarano, Miguel Rodríguez-Piñero Royo y Adrián Todolí Signes (coordinadores), Cambiando la forma de trabajar y de vivir: De las plataformas a la economía colaborativa real (pp. 61-80). Valencia: Tirant lo Blanch.

Arendt, Hannah (2009). Eichmann em Jerusalém. São Paulo: Companhia das Letras.

Doneda, Danilo (2019). Da privacidade à proteção de dados pessoais: Elementos da formação da Lei Geral de Proteção de Dados. São Paulo: Thompson Reuters.

Emerick, Helena, Cláudio Jannotti y Lorena Vasconcelos (2021). «Discriminação algorítmica no trabalho digital». Revista de Direitos Humanos e Desenvolvimento Social, 1: 1-21. Disponible en https://bit.ly/3osxdo6.

FEM, Foro Económico Mundial (2020). Diversity, Equity and Inclusion 4.o: A toolkit for leaders to accelerate social progress in the future of work. Ginebra: World Economic Forum. Disponible en https://bit.ly/3oKLPs1.

Mackenzie, Ewan y Alan McKinlay (2020). «Hope labour and the psychic life of cultural work». Human Relations, 74 (11):1.841-1.863. DOI: 10.1177/0018726720940777.

OIT, Organización Internacional del Trabajo (2019). Telework in the $21^{\mathrm{sT}}$ century: An evolutionary perspective. Northampton: Edward Elgar Publishing. Disponible en https://bit.ly/318hDhy.

PASQuale, Frank (2015). The black box society: The secret algorithms that control money and information. Cambridge: Harvard University Press. Disponible en https:// bit.ly/3otu $48 \mathrm{k}$.

PrassL, Jeremias (2019). «What if your boss was an algorithm? Economic incentives, legal challenges, and the rise of artificial intelligence at work». Comparative Labour Law and Policy Journal, 41: 1-30. Disponible en https://bit.ly/3G66Opg.

RodríGUEZ-PiÑERO, Miguel (2020). «Trabajo en plataformas digitales y regulación: ¿Una respuesta colectiva?». En Macarena Hernández Bejarano, Miguel Rodríguez-Piñero Royo y Adrián Todolí Signes (coordinadores), Cambiando la forma de trabajar y de vivir: De las plataformas a la economía colaborativa real (pp. 147196). Valencia: Tirant lo Blanch.

SAndvig, Christian, Kevin Hamilton, Karrie Karahalios y Cedric Langbort (2016). «When the algorithm itself is a racist: Diagnosing ethical harm in the basic components of software». International Journal of Communication, 10: 4.972-4.990. Disponible en https://bit.ly/3lRAvlD.

SCherTEL, Laura y Marcela Mattiuzzo (2019). «Discriminação algorítmica: Conceito, fundamento legal e tipologia». Revista Direito Público, 16 (90): 39-64. Disponible en https://bit.ly/3pmDZPd.

Schwab, Klaus (2016). The Fourth Industrial Revolution. Ginebra: World Economic Forum. 


\section{Reconocimientos}

Este artículo se presenta como resultado de los diálogos del Grupo de Investigación Trabalho, Seguridade Social e Processo: Diálogos e críticas inscrito en el Directorio de Grupos de Investigación del CNPq.

\section{Sobre los autores}

Helena Emerick Abaurre es estudiante de la Facultad de Derecho de la Universidade Federal do Espírito Santo e investigadora del Grupo de Investigación Trabalho, Seguridade Social e Processo: diálogos e críticas y del Grupo de Investigación Labirinto da Codificação do Direito Processual Civil Internacional. Su correo electrónico es helenaabaurre@gmail.com. (D) https://orcid.org/oooo-0002-7427-9855.

Ricardo José Macedo de Britto Pereira es profesor titular y coordinador académico de la maestría en Relaciones Sociales y Laborales del Centro Universitário do Distrito Federal. Pasantía postdoctoral en Cornell University, Estados Unidos. Doctor por la Universidad Complutense de Madrid. Maestría en Derecho de la Universidad de Syracuse, Estados Unidos. Maestría de la Universidade de Brasilia. Líder del Grupo de Investigación Trabalho, Constituição e Cidadania. Subprocurador general del trabajo jubilado. Su correo electrónico es rjmbpereira@gmail.com.

https://orcid.org/oooo-0003-4510-8894.

Cláudio Jannotti da Rocha es profesor asociado en la Universidad Federal de Espírito Santo. Estudiante de posdoctorado en la Universidade Federal da Bahia. Doctor y máster en Derecho y Proceso Laboral por la Pontifícia Universidade Católica de Minas Gerais. Miembro de la Facultad de Derecho de la Universidade Federal do Espírito Santo. Coordinador del Grupo de Investigación Trabalho, Seguridade Social e Processo: diálogos e críticas. Su correo electrónico es claudiojannotti@hotmail. com. (D) https://orcid.org/0000-0003-2379-2488. 
La Revista Chilena de Derecho del Trabajo y de la Seguridad Social es una publicación semestral del Departamento de Derecho del Trabajo y de la Seguridad Social de la Facultad de Derecho de la Universidad de Chile, y que tiene por objetivo el análisis dogmático y científico de las instituciones jurídico-laborales y de seguridad social tanto nacionales como del derecho comparado y sus principales efectos en las sociedades en las que rigen.

\author{
DIRECTOR \\ Luis Lizama Portal \\ EDITOR \\ Claudio Palavecino Cáceres \\ SECRETARIO DE REDACCIÓN \\ Eduardo Yañez Monje \\ SITIO WEB \\ revistatrabajo.uchile.cl \\ CORREO ELECTRÓNICO \\ pyanez@derecho.uchile.cl \\ LICENCIA DE ESTE ARTÍCULO \\ Creative Commons Atribución Compartir Igual 4.o Internacional
}

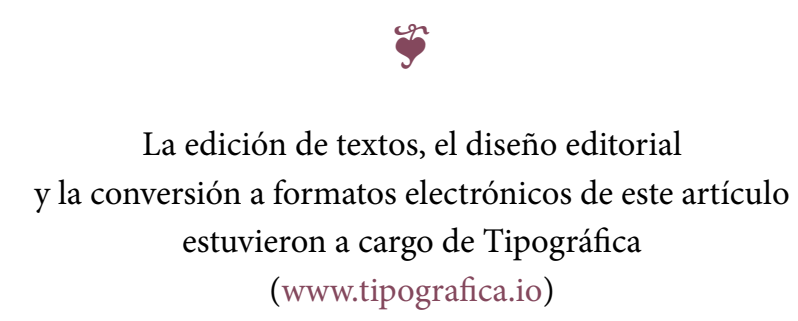

